ORIGINAL ARTICLE

\title{
Role of exercise stress test in master athletes
}

\author{
F Pigozzi, A Spataro, A Alabiso, A Parisi, M Rizzo, F Fagnani, V Di Salvo, G Massazza, N Maffulli
}

Br J Sports Med 2005;39:527-531. doi: 10.1136/bjsm.2004.014340

See end of article for authors' affiliations

....................

Correspondence to: Professor Maffulli, Department of Trauma and Orthopaedic Surgery, Keele University School of Medicine, Thornburrow Drive, Hartshill, Stoke on Trent, Staffordshire ST4 7QB, UK; osal4@ keele.ac.uk

Accepted

27 September 2004

\begin{abstract}
Background: The effectiveness of cardiovascular screening in minimising the risk of athletic field deaths in master athletes is not known.

Objective: To evaluate the prevalence and clinical significance of ST segment depression during a stress test in asymptomatic apparently healthy elderly athletes.

Methods: A total of 113 male subjects aged over 60 were studied ( 79 trained and 34 sedentary); 88 of them ( 62 trained and 26 sedentary) were followed up for four years (mean 2.16 years for athletes, 1.26 years for sedentary subjects), with a resting 12 lead electrocardiogram (ECG), symptom limited exercise ECG on a cycle ergometer, echocardiography, and 24 hour ECG Holter monitoring.

Results: A significant ST segment depression at peak exercise was detected in one athlete at the first evaluation. A further case was seen during the follow up period in a previously "negative" athlete. Both were asymptomatic, and single photon emission tomography and/or stress echocardiography were negative for myocardial ischaemia. The athletes remained symptom-free during the period of the study. One athlete died during the follow up for coronary artery disease: he showed polymorphous ventricular tachycardia during both the exercise test and Holter monitoring, but no significant ST segment depression. Conclusions: The finding of false positive ST segment depression in elderly athletes, although still not fully understood, may be related to the physiological cardiac remodelling induced by regular training. Thus athletes with exercise induced ST segment depression, with no associated symptoms and/or complex ventricular arrhythmias, and no adverse findings at second level cardiological testing, should be considered free from coronary disease and safe to continue athletic training.
\end{abstract}

Abbreviations: $C A D$, coronary artery disease; $E C G$ electrocardiogram; PSVB, premature supraventricular beat; PVB, premature ventricular beat and clinical significance of ST segment depression during stress testing in old, asymptomatic, apparently healthy athletes.

\section{SUBJECTS AND METHODS}

A group of 113 male subjects aged over 60 was studied. Seventy nine (mean (SD) age 62.4 (0.3) years, weight 75.7 (1.0) $\mathrm{kg}$, height $173.1(1.2) \mathrm{cm}$ ) of them regularly took part in sport (track and field, tennis, swimming, or cycling), and 34 (mean (SD) age 62.1 (0.4) years, weight 76.4 (1.3) kg, height $173.9(0.7) \mathrm{cm}$ ) were sedentary. Forty two of the 79 trained subjects were competitive athletes; 14 of them trained at least 10 hours a week, and the remaining 28 trained between 5-9 hours a week.

We followed up 88 of the subjects (62 athletes and 26 sedentary subjects) for up to four years (range 1-4; mean 2.16 for athletes and 1.26 for sedentary subjects). The follow up was performed on an outpatient basis, planned every 912 months, for both athletes and controls. Follow up was incomplete $(77 \%)$ because of the unavailability of the subjects to be tested again or for interruption of their sport for personal reasons or injuries.

All the subjects underwent the same cardiovascular screening protocol, both at baseline and at follow up, including medical history and physical examination, resting 12 lead ECG, symptom limited exercise ECG on cycle ergometer, echocardiography, and 24 hour ECG Holter monitoring. Recent data suggest a higher prevalence of silent ischaemia sedentary counterparts, ${ }^{16}{ }^{17}$ although a high prevalence of false positive exercise tests among master endurance athletes has also been reported. ${ }^{18}$

The purpose of this study was to determine the diagnostic and prognostic value of abnormal ECG response during exercise testing. In particular, we evaluated the prevalence coronary artery disease (CAD), and, in at least half the cases, ic and apparently healthy subjects. ${ }^{2-6}$ The American tory, physical examination, and symptom limited maximal a moderate to high cardiovascular risk profile for CAD-that

Exercise ECG is the most often used non-invasive test to $\mathrm{CAD}$ in asyptomatic, low isk subjects is controversia hem eligibility to participate in competitive sport. ${ }^{15}$

The effectiveness of implementing cardiovascular screening of master athletes with exercise ECG to minimise the risk of athletic field deaths in this population is not known. 
Table 1 Resting and exercise data for athletes $(n=79)$ and sedentary subjects (controls; $\mathrm{n}=34$ )

\begin{tabular}{|c|c|c|c|c|}
\hline & \multicolumn{2}{|l|}{ Athletes } & \multicolumn{2}{|l|}{ Controls } \\
\hline & Rest & Peak exercise & Rest & Peak exercise \\
\hline $\mathrm{SBP}(\mathrm{mm} \mathrm{Hg})$ & $142.1(1.6)$ & $223.6(3.1) \dagger$ & $137.2(2.3)$ & $208.8(3.8)$ \\
\hline $\mathrm{DBP}(\mathrm{mm} \mathrm{Hg})$ & $88.1(0.9)$ & $92.9(1.5)$ & $89.8(1.5)$ & 95.5 (1.9) \\
\hline Heart rate (beats/min) & $66.4(1.4)^{*}$ & $149.8(2.2)$ & $71.2(2.0)$ & $145.9(3.9)$ \\
\hline Max exercise capacity (W) & - & $199.9(5.1) \dagger$ & - & $154.4(6.2)$ \\
\hline
\end{tabular}

\section{Experimental protocol} Echocardiography

Echocardiographic studies were performed using a commercially available unit (ESA OTE Biomedica AU3 Advanced, Milan, Italy). M mode echocardiograms were derived from two dimensional images and recorded on a strip chart at $50 \mathrm{~mm} / \mathrm{s}$. Chamber dimension and wall thickness were determined from an integrated assessment of $M$ mode and two dimensional echocardiograms as recommended by the American Society of Echocardiography. ${ }^{19}$ Doppler data were used as parameters of left ventricular diastolic function. Mitral valve flow was measured by pulsed Doppler, with the sample volume placed at the centre of the mitral valve plane and the echocardiogram simultaneously recorded at a speed of $100 \mathrm{~mm} / \mathrm{s}$.

\section{Exercise ECG}

Cycle ergometry (STS3; Remco Italy Cardioline, Milan, Italy) was performed in the upright position. The exercise protocol included one minute of unloaded cycling, and progressive loadings of $30 \mathrm{~W}$ every two minutes to exhaustion. During the test, a 12 lead ECG (MedGraphics Corporation, St Paul, Minnesota, USA) was continuously recorded. Arterial pressure was measured at baseline and at the end of each exercise stage using a mercury sphygmomanometer.

The test was stopped:

- if there was a significant ST segment depression associated with precordial ache; or

- the ECG evidenced life threatening arrhythmias; or

- there was an abnormal decrease in heart rate and/or systolic blood pressure during exercise ${ }^{20}$; or

- if the subject reported muscular exhaustion

The ST segment depression was measured at least 6080 milliseconds after the $\mathrm{J}$ point in three consecutive beats using PQ interval as the isoelectric line. An ischaemic response was defined as a flat or downsloping ST segment depression of $1.5 \mathrm{~mm}$ in two or more leads including V5.

\section{4 hour ECG Holter monitoring}

Within two weeks of the cycle ergometry test, all subjects underwent 24 hour Holter recording. Subjects were connected to a two channel ECG recorder and two modified CMI and CM5 chest leads. The ECG signal was analysed using a commercially available computer based system (Cardioline AD 35 TOP). Automatic analysis of arrhythmias was performed first. Recordings were then checked manually by two board certified cardiologists with a special interest in sports and exercise cardiology.

\section{Statistical analysis}

Descriptive statistics were calculated. Data were reported as mean (SEM), unless otherwise specified. Differences in the reported variables between athletes and sedentary subjects were evaluated by the $\chi^{2}$ test and unpaired $t$ tests. Relations between variables were assessed by Pearson product-moment correlation. Significance was set at $\mathrm{p}<0.05$.

\section{RESULTS}

No significant differences were found with regard to age, weight, or systolic and diastolic blood pressure between athletes and sedentary subjects. In particular, the prevalence of systolic hypertension (defined as systolic blood pressure $140 \mathrm{~mm} \mathrm{Hg}$ and diastolic blood pressure $90 \mathrm{~mm} \mathrm{Hg}$ ) was the same in the two groups (table 1).

\section{Resting ECG}

Resting heart rate was significantly lower in athletes than in sedentary subjects (66.4 (1.4) v 71.2 (2.0) beats/min). No significant differences were found with regard to mean PR and QT intervals between the two groups.

Fifty three athletes (67\%) and 25 sedentary subjects (73\%) had normal ECG at rest. A variety of abnormalities were found in the other subjects (table 2).

\section{Echocardiography}

End diastolic interventricular septal $\left(\begin{array}{lllll}9.4 & (0.1) & v & 8.1\end{array}\right.$ $(0.3) \mathrm{mm})$ and left ventricular posterior wall $(9.1(0.1) \mathrm{v}$ $8.0(0.3) \mathrm{mm})$ thickness were significantly greater in athletes than in sedentary subjects $(\mathrm{p}=0.03)$. No significant differences were found between the two groups with regard to left ventricular end diastolic internal diameter, left atrial size, and aortic root dimension.

There were no differences in left ventricular diastolic function, evaluated by pulsed wave Doppler pattern of transmitral inflow, between athletes and untrained subjects $(1.09(0.1) \vee 1.20(0.3))$.

Table 2 Electrocardiographic data at rest in athletes $(n=79)$ and sedentary subjects $(n=34)$

\begin{tabular}{lllllllll}
\hline & Normal & LAD $<-30^{\circ}$ & LAD $>-30^{\circ}$ & RBBB & AVB I & AVBII & PVB & VRA \\
\hline Athletes & $26(33)$ & $13(16)$ & $2(2)$ & $3(4)$ & $4(6)$ & $1(1)$ & $3(4)$ & $2(2)$ \\
Sedentary subjects & $9(26)$ & $3(9)$ & 0 & $1(3)$ & 0 & 0 & $3(9)$ & $2(6)$ \\
\hline
\end{tabular}

Values in parentheses are percentages. One athlete showed VRA with a slight LAD. One athlete showed increased PR interval duration with slight LAD. One athlete showed RBBB with distinct LAD.

$L A D<-30$, Left $Q R S$ axis deviation less than $-30^{\circ}$; $L A D>-30^{\circ}$, left $Q R S$ axis deviation more than $-30^{\circ}$; RBBB, right bundle branch block; $A V B$ I, first degree atrioventricular block; AVB II, second degree atrioventricular block; PVB, premature ventricular beats; VRA, ventricular repolarisation abnormalities. 
Table 3 Twenty four hour electrocardiographic Holter monitoring in athletes $(n=60)$ and sedentary subjects $(n=25)$

\begin{tabular}{lllllll}
\hline & imPVBs & ipPVBs & ripPVBs & mPSVBs & PSVBs rip = 3 & PSVBs rip $>3$ \\
\hline Athletes & $35(58)$ & $8(13)$ & $4(7)$ & $31(52)$ & $13(22)$ & $10(17)$ \\
Sedentary & $10(40)$ & $4(16)$ & $5(20)$ & $11(44)$ & $5(20)$ & $5(20)$ \\
subjects & & & & \\
\hline
\end{tabular}

\section{Exercise test}

One athlete developed an unsustained ventricular tachycardia during the cycle ergometer test at $60 \mathrm{~W}$; the test was stopped. All the other subjects ended the exercise test for muscular exhaustion without any symptoms. Athletes were able to reach significantly higher workloads than sedentary subjects $(199.9$ (5.1) v 154.4 (6.2) W; p $=0.01$ ). At peak exercise, systolic BP was higher in athletes than in sedentary subjects (223.6 (3.1) v 208.8 (3.8) $\mathrm{mm} \mathrm{Hg;} \mathrm{p} \mathrm{=} \mathrm{0.01).} \mathrm{No}$ significant differences were found between the two groups with regard to diastolic blood pressure (92.9 (1.5) $v 95.5$ (1.9) $\mathrm{mm} \mathrm{Hg}$ ) and heart rate (149.8 (2.2) v 145.9 (3.9) beats/ $\min )$.

Isolated premature supraventricular beats (PSVBs) were recorded during the exercise test in $12(16 \%)$ athletes and in $21(21 \%)$ sedentary subjects. Two (3\%) athletes had complex PSVBs.

Twenty three (30\%) athletes and $10(36 \%)$ sedentary subjects had isolated, monomorphous premature ventricular beats (PVBs), four (5\%) athletes and one (3\%) sedentary subject had isolated, polymorphous PVBs, five $(6 \%)$ athletes and four $(12 \%)$ sedentary subjects had couples or tachycardias.

A significant ST segment depression at peak exercise was detected in one athlete who denied any symptoms. A further episode of ST segment depression was registered during the follow up period in a previously "negative" athlete.

\section{4 hour ECG Holter monitoring}

PSVBs were recorded in $54(90 \%)$ athletes and 21 (84\%) sedentary subjects. Isolated PSVBs were found in 31 athletes $(52 \%)$ and 11 sedentary subjects $(40 \%)$. Runs of supraventricular tachycardia with no more than three consecutive beats were found in 13 (22\%) athletes and five (24\%) sedentary subjects, and runs of supraventricular tachycardia with more than three consecutive beats were found in 10 $(17 \%)$ athletes and five $(20 \%)$ sedentary subjects.

Ventricular tachyarrhythmias were registered in 47 (78\%) athletes and $19(76 \%)$ sedentary subjects. Isolated, monomorphous PVBs were found in 35 (58.3\%) athletes and 10 $(40 \%)$ sedentary subjects; isolated polymorphus PVBs were found in eight athletes (13\%) and four sedentary subjects (16\%).

Complex ventricular tachyarrhythmias were found in four $(7 \%)$ athletes and five $(20 \%)$ sedentary subjects.

Table 2 summarises ECG data, and table 3 summarises Holter monitoring data.

\section{Follow up}

Two athletes died during the follow up period: one of them from a non-cardiovascular cause, the other from CAD. In the latter, we detected polymorphous ventricular tachycardia both during the exercise test and the 24 hour Holter monitoring, with no significant ST segment depression. We had suggested that this athlete refrain from vigorous physical activity.

\section{DISCUSSION}

Exercise can reduce cardiovascular risk factors and decrease the overall incidence of heart disease. ${ }^{19}{ }^{20}$ Conversely, physical inactivity and sedentary lifestyle are among the major risk factors for the development of CAD..$^{21-23}$

Over the past decade, in Western countries, an increasing number of middle aged or older people have engaged in organised competitive sports, including extremely strenuous disciplines. Overall, exercise related sudden cardiac deaths are uncommon, although dramatic, events in the general athletic population. Epidemiological data suggest that these events are significantly more common in older ( $>35$ years of age) than young athletes with an estimated risk of about $1 / 15000$ to $1 / 18000 \mathrm{v} 1 / 100000$ to $1 / 300000 .^{24}$

The cause of sudden death in the vast majority of older athletes is CAD, which, based on available postmortem data, usually consists of severe $(>75 \%)$ narrowing of the cross sectional area by atherosclerotic plaque in one or more of the major extramural coronary arteries. Two main mechanisms are generally considered to underlie exercise related sudden death: coronary spasm and plaque rupture. ${ }^{25}$ Indeed, although exercise dilates normal coronaries, it may induce coronary spasm in atherosclerotic segments. High shear stress caused by physical activity, increased systolic blood pressure, and contraction of non-compliant atherosclerotic plaque may all trigger plaque disruption and rupture. Finally, sudden death could result from ischaemia induced ventricular fibrillation, even in subjects with non-occlusive atherosclerotic lesions.

The goal of preparticipation cardiovascular screening is to identify athletes with unsuspected cardiac disease, and disqualify them from training and competition to minimise the risk of athletic field deaths. ${ }^{26}$ For master athletes, the screening protocol should be specifically addressed to the identification of CAD. To date, however, the identification of the most useful and cost effective preparticipation screening for these athletes remains an unresolved problem. ${ }^{26}$

Exercise ECG has good sensitivity and specificity for CAD screening in subjects with high pretest probability of disease because of the presence of two or more coronary risk factors. In contrast, exercise ECG has low diagnostic accuracy in low risk populations. ${ }^{27}{ }^{28}$ Moreover, the exercise test has low sensitivity for predicting future coronary events in asymptomatic subjects, even though the predictive power of an abnormal stress test may well be independent of other risk factors for CAD. ${ }^{29}$

Master athletes overall are a very low risk group because the vast majority of them lack the main "environmental" coronary risk factors: smoking, obesity, hypertension, dyslipidaemia, and sedentary life style. Also, available data suggest a higher incidence of false positive tests in athletes than in matched sedentary subjects. ${ }^{30}$

Hood and Northcote ${ }^{18}$ reported a $15.8 \%$ prevalence of false positive ST segment depression in highly trained veteran endurance athletes. Katzel et $a^{16}$ found a $13 \%$ prevalence of false positive ST segment depression in 70 master athletes 


\section{What is already known on this topic}

Acute vigorous physical exertion can trigger adverse cardiovascular events in older, apparently healthy athletes. Most can be ascribed to latent coronary artery disease. The American Heart Association recommends preparticipation screening for men over 40 and women over 50 or postmenopausal, with one or more independent coronary risk factors.

and $6 \%$ in 85 untrained subjects. We ourselves found a false positive ST segment depression in the $2.6 \%$ of the athletes, and in none of the sedentary subjects. Hence, false positive ST segment depression seems to occur more often in elderly athletes than in age matched sedentary subjects. This phenomenon, although still not understood, may be related to the physiological cardiac hypertrophy or the excessive pressure response to exercise observed in such subjects. However, the athletes who showed ST segment depression during stress testing in our study had no left ventricular hypertrophy or hypertensive response to exercise. In addition, they were asymptomatic, and single photon emission tomography and stress echocardiography proved negative for myocardial ischaemia. Finally, during the follow up period they remained free from symptoms and cardiovascular events.

These observations carry practical implications. In fact, although the causes of these false positive ECG alterations in elderly healthy athletes remain unknown, they are often encountered. These subjects should be investigated using second level tests or stress ECG. If these tests do not clarify the diagnosis, coronary angiography should be considered. We suggest that the athletes who are negative after second level tests should be considered free from coronary disease, and they should be safe to compete.

The athlete who died suddenly during the follow up period had not been considered safe to compete because of the development of a non-sustained ventricular tacharrhythmia during the exercise test. Although the arrhythmia developed in the absence of ST segment alteration, we considered it an ischaemic equivalent. Exercise induced myocardial ischaemia, in fact, may trigger ventricular arrhythmias, which in turn could be the cause of sudden death. ${ }^{31}$

Exercise induced complex ventricular arrhythmias, although asymptomatic and not associated with ST segment alteration, represent another critical point of the cardiovascular evaluation of elderly athletes.

Vigorous exercise training is not associated with prevalence of ventricular arrhythmias in elderly athletes in the absence of underlying heart disease. The incidence of ventricular arrhythmias is not different in elderly athletes and nonathletes. In symptom-free athletes, it is unlikely that infrequent PVBs are a pathological finding that would precipitate life threshold arrhythmias. ${ }^{32} 33$ Hence, elderly athletes with evidence of frequent and/or complex ventricular arrhythmias during exercise ECG should be carefully investigated for a potential underlying cardiac disease, particularly ischaemic heart disease, and undergo second level tests, as well as athletes with exercise induced ST segment alteration.

The main limitation of this study is the relative short follow up and the fact that nearly $25 \%$ of subjects did not perform a further exercise test. The long term significance of ST segment depression in asymptomatic athletes remains unknown, and this study highlights the efficacy of a stress test as a simple, effective screening method for detection of cardiovascular abnormalities in elderly athletes.
What this study adds

Athletes with exercise induced ST segment depression, with no associated symptoms and/or complex ventricular arrhythmias, and no adverse findings at second level cardiological testing, should be considered free from coronary disease and safe to continue athletic training.

In conclusion, exercise ECG in master athletes can minimise the risk of sudden death. We suggest that an ST segment depression, even in the absence of subjective symptoms, should be considered suspicious, and investigated. However, when false positivity is well documented, the athlete should be safe to compete. Subjects with "positive" exercise test (ST segment depression and/or complex ventricular arrhythmias) should be considered at risk of exercise induced cardiac events and submitted to second level examination.

\section{Authors' affiliations}

F Pigozzi, A Spataro, A Alabiso, A Parisi, M Rizzo, F Fagnani, V Di Salvo, G Massazza, Sports Medicine Unit, University Institute of Movement Sciences, Rome, Italy

N Maffulli, Department of Trauma and Orthopaedic Surgery, Keele University School of Medicine, Stoke on Trent, Staffordshire, UK

Competing interests: none declared

\section{REFERENCES}

1 Maron BJ, S. Arau'Jo CG, Thompson PD, et al. Recommendations for prepartecipation screening and the assessment of cardiovascular disease in master athletes. An advisory for healthcare professional from the working groups of the World Heart Federation, the International Federation of Sports medicine, and the American Heart Association Committee on Exercise, Cardiac Rehabilitation, and Prevention. Circulation 2001;103:327-34.

2 Maron BJ, Epstein SE, Roberts WC. Causes of sudden death in competitive athletes. J Am Coll Cardiol 1986:7:204-14.

3 Ciampricotti R, El Gamal M. Recurrent myocardial infarction and sudden death after sport. Am Heart J 1989; 117:188-91.

4 Ciampricotti R, El Gamal M, Bonnier JJ, et al. Myocardial infarction and sudden death after sport: acute coronary angiographic findings. Cathet Cardiovasc Diagn 1989;17:193-7.

5 Opie LH. Sudden death and sport. Lancet 1975;1:263-6.

6 Thompson PD. Athletes, athletics, and sudden cardiac death. Med Sci Sports Exerc 1993:981-4.

7 Gibbson RJ, Balady GJ, Beasley JW, et al. ACC/AHA guidelines for exercise testing: a report of the American College of Cardiology/American Heart Association Task Force on practice guidelines (commitee on exercise testing) J Am Coll Cardiol 1997;30:260-315.

8 Spin JM, Prakash M, Froelicher VF, et al. The prognostic value of exercise testing in elderly men. Am J Med 2002;1 12:453-9.

9 Goraya TY, Jacobsen SJ, Pellikka PA, et al. Prognostic value of treadmill exercise testing in elderly persons. Ann Intern Med 2000;132:862-70.

10 Rywik TM, Zink RC, Gittings NS, et al. Independent prognostic significance of ischemic ST-segment response limited to recovery from treadmill exercise in asymptomatic subjects. Circulation 1998;97:2117-22.

11 Gibbons LW, Mitchel TL, Wei M, et al. Maximal exercise test as a predictor of risk for mortality from coronary heart disease in asymptomatic men. Am J Cardiol 2000;86:53-8.

12 Frame PS. A critical rewiew of adult health maintenance. I. Prevention of atherosclerotic diseases. J Fam Pract 1986;22:341-6.

13 Breslow L, Somers AR. The lifetime health-monitoring program: a practical approach to preventive medicine. N Engl J Med 1977;296:601-8.

14 Smith RH, LePetri B, Moisa RB, et al. Association of increased left ventricular mass in the absence of electrocardiographic left ventricular hypertrophy with ST depression during exercise. Am J Cardiol 1995:76:973-4.

15 Società Italiana di Cardiologia dello sport (SIC Sport). Protocolli cardiologici per il giudizio di idoneità allo sport agonistico 2003. Rome: Casa Editrice Scientifica Internazionale, 1-210.

16 Katzel LI, Fleg J, Busby-Whitehead MJ, et al. Exercise-induced silent myocardial ischemia in master athletes. Am J Cardiol 1998;81:261-5.

17 Jensen K, Bouvier F, Saltin B, et al. High prevalence of arrhythmias in elderly male athletes with a lifelong history of regular strenous exercise. Heart 1998;79:161-4.

18 Hood S, Northcote RJ. Cardiac assessment of veteran endurance athletes: a 12 year follow up study. Br J Sports Med 1999;33:239-43.

19 Sahn DJ, De Maria A, Kisslo J, et al. Reccomendation regarding quantitation in M-Mode echocardiography. Circulation 1978;58:1072-83. 
20 ACSM. Resource manual for guidelines for exercise testing and prescription 4th ed. Baltimore: Williams and Wilkins.

21 Berlin J, Colditz A. A meta analysis of physical activity in the prevention of coronary heart desease. Am J Epidemiol 1990;132:612-27.

22 Blair SN, Kampert JB, Kohl HWIII, et al. Physical fitness and incidence of hypertension in healthy normotensive men and women. JAMA 1996;276:205-10.

23 Paffembarger RS, Hyde RT, Wing AL, et al. The association of changes in physical activity level and other lifestyle characteristics with mortality among men. N Engl J Med 1999;328:538-45.

24 Lee IM, Hsieeh CC, Paffembarger RS Jr. Exercise intensity and longevity in men: the Harvard Alumni Health Study. JAMA 1995;273:1179-84.

25 Basilico FC. Cardiovascular disease in athletes. Am J Sports Med 1999;27:108-21.

26 Maron BJ, Epstein SE, Roberts WC. Causes of sudden death in competitive athletes. J Am Coll Cardiol 1986;7:204-14.
27 Pigozzi F, Spataro A, Fagnani F, et al. Preparticipation screening for the detection of cardiovascular abnormalities that may cause sudden death in competitive athletes. Br J Sports Med 2003;37:4-5

28 Detramo R, Froelicher $\mathrm{V}$. A logical approach to screening for coronary artery disease. Ann Int Med 1987;106:846-52.

29 Sheffield L. Exercise stress testing for coronary artery disease. In: Braunwald E, ed. Heart disease. A texbook of cardiovascular medicine. 3rd ed. Philadelphia: WB Saunders, 1988:223-41.

30 Fortuin NJ, Weiss JL. Exercise stress testing. Circulation 1977:56:699-712.

31 Blair SN, Kohl HW III, Barlow CE, et al. Changes in physical fitness and allcause mortality: a prospective study of healthy and unhealthy men. JAMA 1995;273:1093-8.

32 Noakes TD. Heart disease in marathon runners: a review. Med Sci Sports Exerc 1987; 19:187-94.

33 Pigozzi F, Alabiso A, Parisi A, et al. Vigorous exercise training is not associated with prevalence of ventricular arrhythmias in ederly athletes. J Sports Med Phys Fitness 2004;44:92-7.

\section{$\mathrm{ECHO}$}

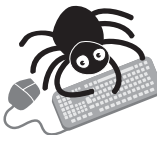

Please visit the British Journal of Sports Medicine website [www. bisportmed. com] for a link to the full text of this article.

\section{Physiological upper limits of ventricular cavity size in highly trained adolescent} athletes

J Makan, S Sharma, S Firoozi, G Whyłe, P G Jackson, W J McKenna

Objectives: To define physiological upper limits of left ventricular (LV) cavity size in trained adolescent athletes.

Design: Cross sectional echocardiographic study.

Setting: British national sports training grounds and Olympic Medical Institute.

Subjects: 900 elite adolescent athletes (77\% boys) aged 15.7 (1.2) years participating in ball, racket, and endurance sports and 250 healthy controls matched for age, sex, and size.

Main outcome measures: LV end diastolic cavity size.

Results: Compared with controls, athletes had a larger LV cavity (50.8 (3.7) v 47.9 (3.5) $\mathrm{mm})$, a difference of $6 \%$. The LV cavity was $>54 \mathrm{~mm}$ in $18 \%$ athletes, whereas none of the controls had an LV cavity > $54 \mathrm{~mm}$. The LV cavity exceeded predicted sizes in $117(13 \%)$ athletes. Among the athletes with LV dilatation, 78\% were boys, LV size ranged from 52-60 $\mathrm{mm}$, and left atrial diameter and LV wall thickness were enlarged. Systolic and diastolic function were normal. None of the athletes in the study had an LV cavity size $>60 \mathrm{~mm}$. LV cavity size correlated with age, sex, heart rate, and body surface area.

Conclusion: Highly trained junior athletes usually have only modest increases in LV cavity size. A proportion of trained adolescent athletes have LV cavity size exceeding predicted values but, in absolute terms, LV cavity rarely exceeds $60 \mathrm{~mm}$ as in patients with dilated cardiomyopathy. In highly trained adolescent athletes with an LV cavity size $>60 \mathrm{~mm}$ and any impairment of systolic or diastolic function, the diagnosis of dilated cardiomyopathy should be considered.

\ Heart 2005;91:495-499. 